\title{
INFLUENCE OF UNCERTAINTY IN AERODYNAMIC PERFORMANCE ON THE DYNAMIC RESPONSE OF A TWO STAGE GEAR SYSTEM
}

\author{
Manel Tounsi, Moez Beyaoui, Kamel Abboudi, Nabih Feki, \\ Lassaad Walha, Mohamed Haddar \\ Laboratory of Mechanics, Modelling and Mansufacturing (LA2MP), National School of Engineers of Sfax, Sfax, Tunisia \\ e-mail: manelt@yahoo.fr; moez.beyaoui@yahoo.fr; kamalo1982@yahoo.fr; fekinabih@gmail.com; walhalassaad@yahoo.fr; \\ mohamed.haddar@enis.rnu.tn
}

\begin{abstract}
In this paper, the nonlinear dynamic response in a wind turbine system is considered and the quantification of uncertainty effects on the variability of this nonlinear response is investigated. Under dynamic conditions, a lumped model with 12 degrees of freedom is proposed taking into account the uncertainty associated to the power coefficient of the input aerodynamic torque. The dynamic response of the two-stage spur gear system is obtained using ODE45 solver of Matlab. The Polynomial Chaos (PC) method is used to introduce the uncertainties on the proposed model. A comparison between the two dynamic responses given by the proposed lumped dynamic model takes into account the uncertainty. It is performed on the existed model without uncertainty. Thus, the efficiency and robustness of the proposed new methodology is evaluated.
\end{abstract}

Keywords: gearbox, uncertainty, power coefficient, random parameter, polynomial chaos

\section{Introduction}

Recently, due to increasing demand for energy, there has been a rapid development of wind turbines all over the world. This constant growth in energy consumption and polluting effects associated are in the heart of the issue of the environmental care, so that an increasing attention is being paid to wind energy. Generally, wind turbines are one of the machines that take advantage of wind energy to generate electrical power.

During preliminary design of dynamic systems, many physical parameters can have a significant effect on the vibration response of the system. Indeed, some features can generate nonlinear responses need to be taken into account. The aerodynamic complexities are involved in optimisation of wind turbine systems in an attempt to maximise its performance. Their aerodynamic and dynamic properties have a decisive influence on the entire system. These properties are responsible of rotor capability to convert wind energy into mechanical energy. Thus, the overall efficiency of the energy conversion in the wind turbine is determined.

Several studies have been developed to study the dynamic behaviour of wind turbines (Abboudi et al., 2011; Helsen et al., 2011; Zhu et al., 2014). However, the modelling of these mechanical systems admits strong dispersions and uncertainties. In this context, design parameters may vary in an uncertain way during the manufacturing monitoring or operation. Thus, the response may change in some uncertain way. Therefore, the formulation of dynamical systems requires introducing uncertainties into input parameters. In this field, Wei et al. (2015) studied the dynamic response of a geared transmission system of a wind turbine with uncertainty.

To take into account the uncertainties, different methods are reported in the literature, such as Monte Carlo simulations (Rubinstein, 1981; Kalos and Whitlock, 1986), Polynomial Chaos Expansion (Wiener, 1938; Ghanem and Spanos, 1991; Fisher and Bhattacharya, 2008). 
The main idea of the polynomial chaos methods is to transform the stochastic differential equations by means of an intrusive Galerkin projection (Ghanem and Spanos, 1991; Jakerman and Roberts, 2009) into a deterministic set of differential equations. Moreover, mechanical systems operate under parametric and external excitation uncertainties. Such as reported in the literature, the Polynomial Chaos approach is the efficient method comparing to the Monte Carlo approach for quantifying the effects of such uncertainties on the system response.

The capabilities of polynomial chaos have been illustrated in numerous fields, such as environmental and biological problems (Isukapalli et al., 1998a,b), fluid dynamics (Pettersson et al., 2009; Chantrasmi et al., 2066), multibody dynamic systems (Sandu et al., 2006a,b).

In this study, the main originality is that the treatment of uncertainties in the dynamic analysis of a wind turbine system is proposed. The dynamic behaviour of nonlinear systems is investigated in order to analyse the robustness and reliability. For that, a dynamic lumped model of a two-stage gear system is developed in this paper. Three-bladed horizontal-axis wind turbines are considered with 12 degrees of freedom in the presence of the aerodynamic torque that is highlighted by an uncertain coefficient of performance belonging to a well-defined interval. Finally, the main goal of this work is to determine the dynamic behaviour of the gearbox transmission system of the wind turbine generated by uncertainty parameters.

\section{Dynamic modelling}

The studied system is a wind turbine. The increased speed mechanism is a two-stage gear system. It is composed by two trains of gearings supposed without manufacturing defects. In order to make this system more reliable, resistant and sustainable, a numerical analysis of the mechanical system is developed to study the dynamic response.

Figure 1 shows the dynamic model of the two stages gear system. The power transmission of the wind turbine is composed of the two-stage spur gear system. It is presented by three main blocks. The first block $(j=1)$ includes wheel 11 representing the turbine, main shaft and gear 12. The second block $(j=2)$ includes gear 21 , intermediate flexible shaft and gear 22 . Finally, the third block $(j=3)$ is composed by gear 31 , intermediate shaft and wheel 32 which is the representative wheel of the electrical generator.

Every block $j$ is supported by a flexible bearing having two stiffnesses: the bending stiffness $k_{x j}$ and the traction-compression stiffness $k_{y j}$. Each intermediate flexible shaft has a negligible mass compared to the turbine and the generator. It admits some torsional stiffness $k_{\theta j}$. Wheels (11) and (32) characterise respectively the motor side (inertia $I_{11}$ ) and the receiving side (inertia $I_{32}$ ). Angular displacements of each wheel about their rotation axes are denoted by $\theta_{j i}$. The indices $j=1$ to 3 designates the number of the block and the indices $i=1$ to 2 designates the two wheels of each block.

Besides, the linear displacements of the bearing denoted by $x_{j}$ and $y_{j}$ are measured in the plane which is orthogonal to the axes of rotation of the wheels. Each pair of wheels is linked through flexible teeth. This flexibility causes displacements. The gear-mesh contacts are modelled by a linear time varying stiffness $k(t)$ along the lines of action in the spur gear stage.

The gear mesh stiffness can be modelled by a sinusoid wave or by a square wave depending on the type of gear employed (for spur gear the stiffness function is a square wave, for helical gear it is a sinusoid wave function). So the periodic square wave form is the most representative for description of operation of gear systems (Fig. 2).

The terms $\varepsilon_{\alpha}$ are the contacts ratio corresponding to the two gear mesh contacts and $T_{e}$ is the mesh period.

The teeth deflection, denoted by $\delta_{i}(t)$, is projected along the line of action because the gear mesh stiffness is defined along this direction. 


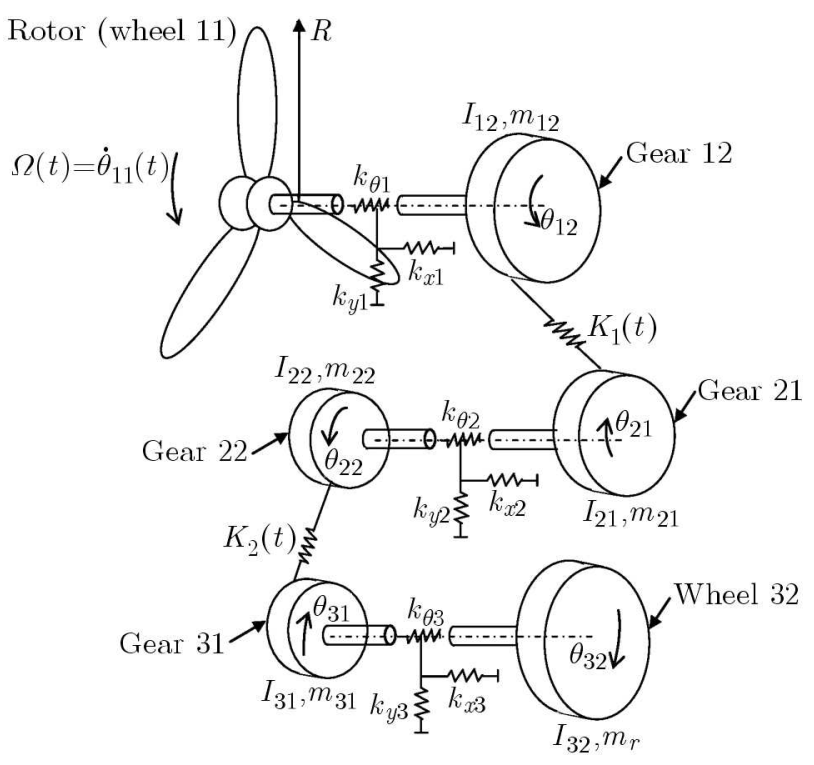

Fig. 1. Components of the wind turbine system

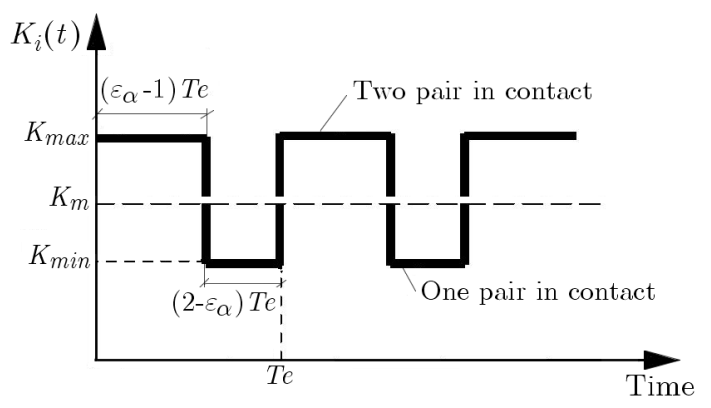

Fig. 2. Modelling of the mesh stiffness fluctuation

The first deflection $\delta_{1}(t)$ along the first gear-mesh contact is given by

$$
\delta_{1}(t)=\left(x_{1}-x_{2}\right) \sin \alpha_{1}+\left(y_{1}-y_{2}\right) \cos \alpha_{1}+r b_{12} \theta_{12}+r b_{21} \theta_{21}
$$

while the deflection $\delta_{2}(t)$ can be written by

$$
\delta_{2}(t)=\left(x_{2}-x_{3}\right) \sin \alpha_{2}+\left(-y_{2}+y_{3}\right) \cos \alpha_{2}+r b_{22} \theta_{22}+r b_{31} \theta_{31}
$$

while $\alpha_{n}$ represents the pressure angle (generally equal to $20^{\circ}$ ) and $r b_{j i}$ are the base radii of the wheels.

\section{Aerodynamic torque}

The maximisation of the power coefficient presents a fundamental role in the wind turbine design to optimise the extraction of energy and to increase the efficiency (Beltran et al., 2011; Buckspan, 2012). The power coefficient is defined by the ratio of power available on the primary shaft and the power of wind. The optimum design of the aerodynamic unit of a wind turbine can be achieved from considering uncertainty of the power coefficient.

For the wind turbine system studied in this paper, we consider that the rotor is composed of three blades removed by an angle of $120^{\circ}$ (Gebreslassie et al., 2013) and connected by a hub, which houses the system for regulating the angular speed. 
The rotor is presented by wheel (11) rotating with some angular velocity and have an input aerodynamic torque to the power transmission system such as shown in Fig. 1.

Sloth et al. (2011) considered that the power in the wind depends on the wind speed, air density, and the swept area. Here, the aerodynamic torque is expressed by the following equation (Lei et al., 2013)

$$
C_{\text {aero }}=\rho_{\text {air }} A R^{3} \Omega^{2} C_{p}
$$

where $\rho_{\text {air }}$ represents the air density, $A$ and $R$ are the area and the radius of the rotor, respectively, $\Omega$ is the angular velocity and $C_{p}$ is the power coefficient.

The power coefficient for the existing model is assumed deterministic by the following empirical expression (Abboudi et al., 2011)

$$
C_{p}=0.44\left(\frac{125}{\lambda}-6.94\right) \mathrm{e}^{\frac{16.5}{\lambda}}
$$

where $\lambda=\Omega R / V(t)$ and $V(t)$ is the wind velocity.

\section{Formulation of equations of motion}

The Lagrange formalism leads to the set of differential equations governing the system motion

$$
\mathbf{M} \ddot{\mathbf{X}}+\left(\mathbf{K}_{s}+\mathbf{K}(t)\right) \mathbf{X}=\mathbf{F}(C p)
$$

The generalised vector of coordinates $\mathbf{X}$ is defined by

$$
\mathbf{X}(t)=\left[x_{1}, y_{1}, x_{2}, y_{2}, x_{3}, y_{3}, \theta_{11}, \theta_{12}, \theta_{21}, \theta_{22}, \theta_{31}, \theta_{32}\right]^{\mathrm{T}}
$$

The matrix $\mathbf{M}$ representing the global mass matrix is expressed by

$$
\begin{aligned}
& \mathbf{M}=\left[\begin{array}{cc}
\mathbf{M}_{L} & \mathbf{0} \\
\mathbf{0} & \mathbf{M}_{A}
\end{array}\right] \\
& \mathbf{M}_{L}=\operatorname{diag}\left(m_{1}, m_{1}, m_{2}, m_{2}, m_{3}, m_{3}\right) \quad \mathbf{M}_{A}=\operatorname{diag}\left(I_{11}, I_{12}, I_{21}, I_{22}, I_{31}, I_{32}\right)
\end{aligned}
$$

where $m_{j}$ is the mass of the block $j$ and $I_{j i}$ is the inertia.

The matrix $\mathbf{K}_{s}$ is the average stiffness matrix of the structure defined by

$$
\mathbf{K}_{s}=\left[\begin{array}{cc}
\mathbf{K}_{p} & \mathbf{0} \\
\mathbf{0} & \mathbf{K}_{\theta}
\end{array}\right]
$$

where $\mathbf{K}_{p}$ represents the bearing stiffness and $\mathbf{K}_{\theta}$ represents the torsional stiffness matrix of shafts

$$
\begin{aligned}
\mathbf{K}_{p}= & \operatorname{diag}\left(k_{x 1}, k_{y 1}, k_{x 2}, k_{y 2}, k_{x 3}, k_{y 3}\right) \\
\mathbf{K}_{\theta}= & {\left[\begin{array}{cccccc}
k_{\theta 1} & -k_{\theta 1} & 0 & 0 & 0 & 0 \\
-k_{\theta 1} & k_{\theta 1} & 0 & 0 & 0 & 0 \\
0 & 0 & k_{\theta 2} & -k_{\theta 2} & 0 & 0 \\
0 & 0 & -k_{\theta 2} & k_{\theta 2} & 0 & 0 \\
0 & 0 & 0 & 0 & k_{\theta 3} & -k_{\theta 3} \\
0 & 0 & 0 & 0 & -k_{\theta 3} & k_{\theta 3}
\end{array}\right] }
\end{aligned}
$$

The matrix $\mathbf{K}(t)$ is the gear mesh stiffness matrix

$$
\mathbf{K}(t)=\mathbf{K}_{m}+\mathbf{K}_{v}(t)
$$


Each gear mesh stiffness variation is approximately modelled by the function $K(t)$. It is composed of an average component $K_{m}$ and a variable component $K_{v}(t)$

$$
K_{v}(t)=\left\{\begin{array}{ll}
K_{\min } & \text { if } t<t_{a} \\
K_{\max } & \text { else }
\end{array} \quad \text { where } \quad t_{a}=\left(2-\varepsilon_{\alpha}\right) T_{e}\right.
$$

The external force vector $\mathbf{F}$ can be written by

$$
\mathbf{F}=\left[0,0,0,0,0,0, C_{\text {aero }}, 0,0,0,0,-C_{r}\right]^{\mathrm{T}}
$$

where $C_{r}$ presents the receiving torque. It is defined by the aerodynamic torque $C_{a e r o}$ divided by the gear ratio $G R$ expressed by

$$
G R=\left(\frac{Z_{12} Z_{22}}{Z_{21} Z_{31}}\right)
$$

\section{Modal analysis and dynamic response}

The technological and dimensional parameters of the two-stage gear system (Abboudi et al., 2011) are summarised in Table 1.

Table 1. System parameters

\begin{tabular}{|l|c|c|c|}
\hline Description & Symbol & Value & Units \\
\hline \hline Gear material density (42CrMo4) & $\rho$ & 7860 & $\mathrm{Kg} / \mathrm{m}^{3}$ \\
\hline Rotor diameter & $D$ & 12 & $\mathrm{~m}$ \\
\hline Bending stiffness & $k_{x j}$ & $7 \cdot 10^{8}$ & $\mathrm{~N} / \mathrm{m}$ \\
\hline Traction stiffness - compression & $k_{y j}$ & $6 \cdot 10^{8}$ & $\mathrm{~N} / \mathrm{m}$ \\
\hline Average mesh stiffness & $k_{m}$ & $2 \cdot 10^{8}$ & $\mathrm{~N} / \mathrm{m}$ \\
\hline Torsional stiffness of the shaft & $k_{\theta j}$ & $5 \cdot 10^{6}$ & $\mathrm{~N} \mathrm{~m} / \mathrm{rad}$ \\
\hline Number of teeth & $Z(12), Z(21)$ & 72,18 & - \\
\cline { 2 - 4 } & $Z(22), Z(31)$ & 54,18 & - \\
\hline Module of teeth & $m$ & 0.016 & $\mathrm{~m}$ \\
\hline Contact ratio & $\varepsilon_{\alpha 1}-\varepsilon_{\alpha 2}$ & $1.67-1.64$ & - \\
\hline
\end{tabular}

In this contribution, the modal analysis focuses on the dynamic properties of system under vibrational excitation is considered. The goal of the modal analysis is to determine the natural mode vibration and frequencies of a structure. Thus, the stiffness matrix of the model is assumed to be the average matrix in order to determine the eigenvalue and modal vibration of the system. The dynamic system response is different at each natural frequency. A null eigenvalue indicates rigid body motion. The dashed lines indicate the initial wheel positions.

Figure 3 represents the reference position and some eigen modes of the two-stage gear system. The fifth mode (mode of pure translation) is relative to the fifth eigen value $w_{p 5}=4600 \mathrm{rad} / \mathrm{s}$. The first mode (mode of pure rotation) characterises the rigid body motion. Finally, the tenth mode is relative to the tenth eigen value $w_{p 10}=40700 \mathrm{rad} / \mathrm{s}$, in fact this mode is a combined mode of translation and rotation.

In order to compare the two models with and without uncertainty, the power coefficient of the aerodynamic torque is considered without uncertainty in this Section. The Newmark method is employed to resolve the equations of motion obtained by the Lagrange formalism.

Figure 4 presents evolution of the displacements of the first (input) and the third (output) bearings. The figures show that the bearing dynamic behaviour is symmetric according to the $y$ direction as a function of the $x$ direction: $y=f(x)$. 


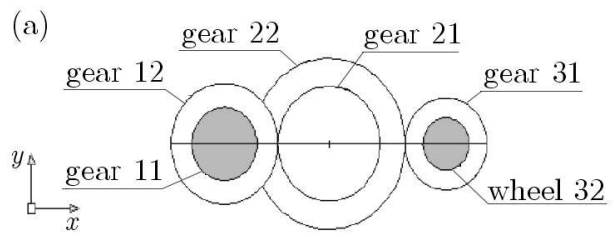

(b)

(c)
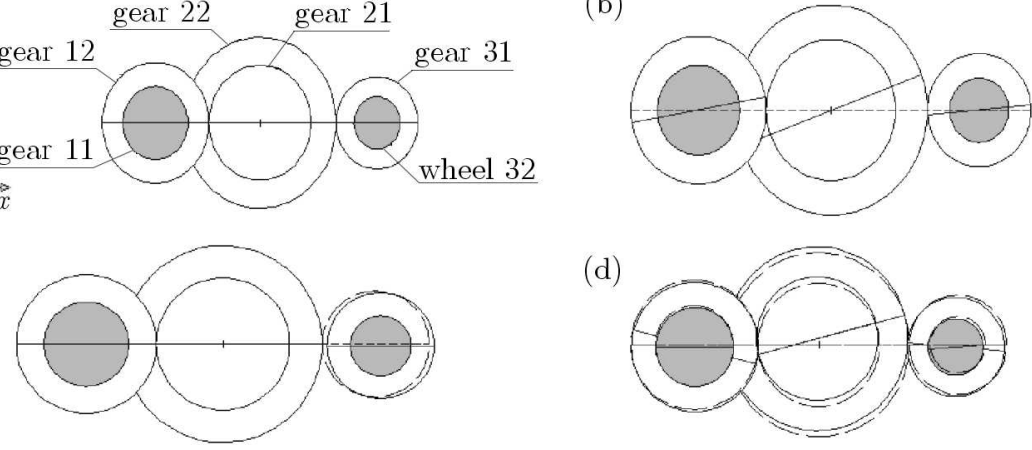

(d)

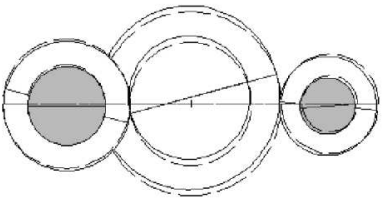

Fig. 3. Mode shapes of the gear system; (a) reference position, (b) pure rotation mode (rigid body motion $),(\mathrm{c})$ pure translation mode $\left(f_{5}=740 \mathrm{~Hz}\right),(\mathrm{d})$ combined mode $\left(f_{10}=6460 \mathrm{~Hz}\right)$
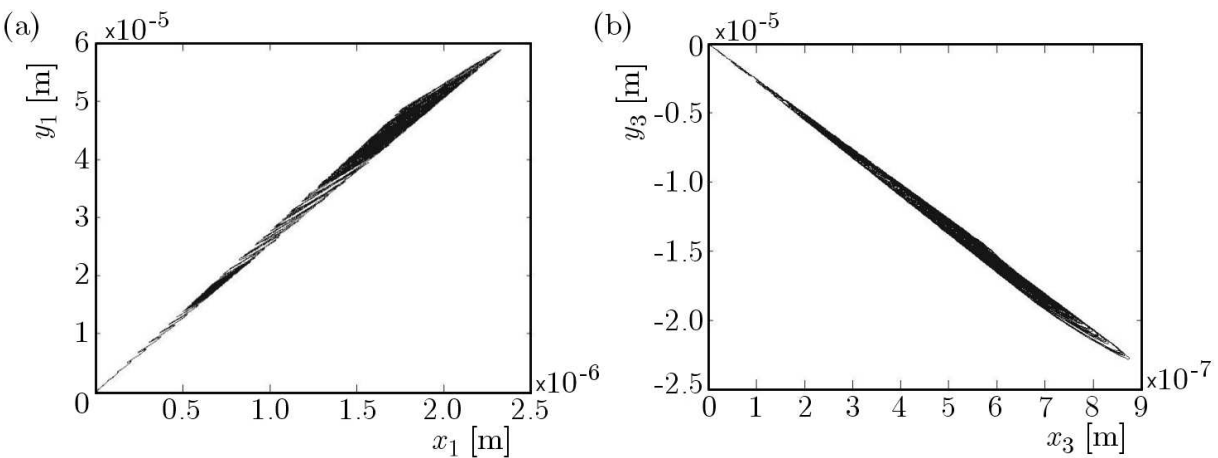

Fig. 4. Displacements of the first and third bearing; (a) first bearing, (b) third bearing

Figure 5 presents the fluctuation of deflections of the first and second tooth. These deflections are due to teeth flexibility. The deflection has an amplitude in the order of $10^{-5} \mathrm{~m}$.
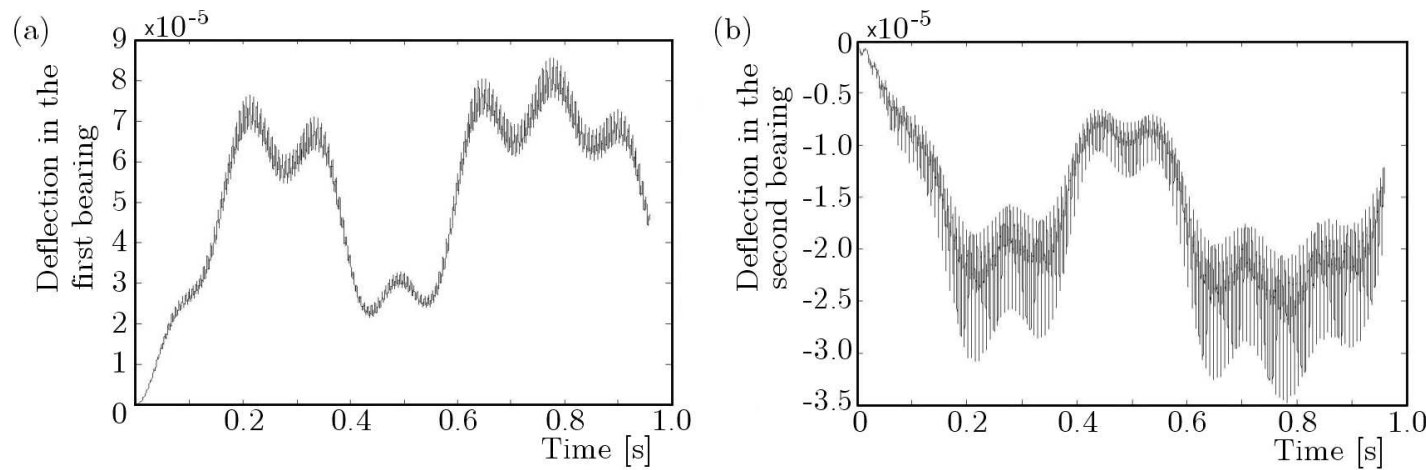

Fig. 5. Fluctuation of deflections in the first and second stage; (a) First stage; (b) second stage

\section{Application of the polynomial chaos method}

The fundamental idea of this approach is to establish a separation between the stochastic components of a random function and its deterministic components. The random process of interest is approximated by sums of orthogonal polynomial chaos of random independent variables. In this context, any uncertain parameter can be viewed as a second order random process. Therefore, the second order random process $z$ can be expanded in terms of orthogonal polynomial chaos as (Nechak et al., 2011) 


$$
z=\sum_{j=0}^{\infty} \bar{z}_{j} \phi_{j}(\xi)
$$

where $\xi$ is a vector of standard normal random variables with the known joint density function $W(\xi), \bar{z}_{j}$ are stochastic modes of the random process $z$ and $\phi_{j}$ are orthogonal polynomial functions satisfying the orthogonally relation

$$
\left\langle\phi_{i} \phi_{j}\right\rangle=\int \phi_{i}(\xi) \phi_{j}(\xi) W(\xi) d \xi
$$

where $\langle\cdot\rangle$ means the internal product operator and $W(\xi)$ is the probability density function (PDF) of random variables that make up the vector $\xi$. The PDF (Xiu and Karniadakis, 2002) acts as a weighting function in the orthogonally relation for $\phi_{j}(\xi)$. Therefore, the type of orthogonal expansion polynomials depends on the nature of the stochastic process through the PDF of the random variables that describe the probability space. In practice, the generalised polynomial chaos expansion is truncated to a finite number of terms $P$. The truncation of the infinite series is necessary to keep the problem computationally feasible. In this work, we will truncate the series in such a way that all expansion polynomials up to a certain maximum degree, denoted by $p$, are included. The number of terms $(P+1)$ in the expansion now follows from this maximum degree $r$ and the dimensionality $n$ of the random vector according to

$$
P=\frac{(r+n) !}{r ! n !}
$$

Then, the computing of $z$ is transformed into the problem of finding the coefficients $\bar{z}_{j}$ of its truncated expansion. The intrusive and non-intrusive approaches are defined to calculate these coefficients called stochastic modes. The non-intrusive approach is shown to be more efficient than the intrusive approach. This approach requires simulations that correspond to particular samples of the random variables and needs no modifications of the stochastic model, contrary to the instructive approach.

The system in this work is equivalently expressed as follows

$$
\mathbf{M} \ddot{\mathbf{Q}}+\mathbf{K}(t) \mathbf{Q}=\mathbf{F}(C p)
$$

A representation in the state space can reduce the order of the system to get a first order system, and it can be written as follows

$$
\dot{\mathbf{q}}(t)=A \mathbf{q}(t)+f(\mathbf{q}(t), C p)
$$

The robust analysis is based on the system representation in the phase space defined by the displacements and velocities

$$
\begin{aligned}
& \mathbf{q}(t)=\left[\theta_{11}, \dot{\theta}_{11}, \theta_{12}, \dot{\theta}_{12}, \theta_{21}, \dot{\theta}_{21}, \theta_{22}, \dot{\theta}_{22}, \theta_{31}, \dot{\theta}_{31}, \theta_{32}, \dot{\theta}_{32},\right. \\
& \left.x_{1}, \dot{x}_{1}, x_{2}, \dot{x}_{2}, x_{3}, \dot{x}_{3}, y_{1}, \dot{y}_{1}, y_{2}, \dot{y}_{2}, y_{3}, \dot{y}_{3}\right]^{\mathrm{T}}
\end{aligned}
$$

The coefficient of performance of the aerodynamic torque is supposed a random variable according to a uniform distribution law defined as follows

$$
C_{p}(\xi)=\frac{b+a}{2}+\frac{b-a}{2} \xi
$$

According to the state of the art, the Legendre polynomials are the best suited to deal with uniform uncertainties. Here $\xi$ is distributed uniformly within the orthogonally interval $[-1,1]$ of the Legendre polynomials. It models the uncertainty of the parameter $C_{p}$ in the interval $[a, b]=[0.35,0.45]$. 
The Legendre polynomials calculated using the recurrence relation are as follows

$$
(n+1) L_{n+1}(x)=(2 n+1) x L_{n}(x)-n L_{n-1}(x) \quad L_{0}(x)=1 \quad L_{1}(x)=x
$$

The decomposing of the random in the Legendre polynomial basis using the Galerkin projection allows generating a non-linear deterministic differential equation system

$$
\begin{aligned}
& \ddot{\bar{\theta}}_{11, l}=-\frac{k_{\theta 1}}{\operatorname{Im}}\left(\bar{\theta}_{11, l}-\bar{\theta}_{12, l}\right)+\frac{1}{\left\langle L_{l}^{2}(\xi)\right\rangle} \frac{\rho A R^{3}}{\operatorname{Im}} \sum_{j=0}^{p} \sum_{k=0}^{p} \dot{\bar{\theta}}_{11, j} \dot{\bar{\theta}}_{11, k}\left\langle C p(\xi), L_{j}(\xi), L_{k}(\xi), L_{l}(\xi)\right\rangle \\
& \ddot{\bar{\theta}}_{12, l}=\frac{k_{\theta 1}}{I_{1}}\left(\bar{\theta}_{11, l}-\bar{\theta}_{12, l}\right)-\frac{R b_{1}}{I_{1}} K_{1}(t) \delta_{1, l} \\
& \ddot{\bar{\theta}}_{21, l}=\frac{k_{\theta 2}}{I_{2}}\left(-\bar{\theta}_{21, l}+\bar{\theta}_{22, l}\right)-\frac{R b_{2}}{I_{2}} K_{1}(t) \delta_{1, l} \\
& \ddot{\bar{\theta}}_{22, l}=\frac{k_{\theta 2}}{I_{3}}\left(\bar{\theta}_{21, l}-\bar{\theta}_{22, l}\right)+\frac{R b_{3}}{I_{3}} K_{2}(t) \delta_{2, l} \\
& \ddot{\bar{\theta}}_{31, l}=\frac{k_{\theta 3}}{I_{4}}\left(-\bar{\theta}_{31, l}+\bar{\theta}_{32, l}\right)+\frac{R b_{4}}{I_{4}} K_{2}(t) \delta_{2, l} \\
& \ddot{\bar{\theta}}_{32, l}=\frac{k_{\theta 3}}{I_{r}}\left(\bar{\theta}_{31, l}-\bar{\theta}_{32, l}\right)-\frac{1}{\left\langle L_{l}^{2}(\xi)\right\rangle} \frac{\rho A R^{3}}{I r} \\
& \sum_{j=0}^{p} \sum_{k=0}^{p} \dot{\bar{\theta}}_{11, j} \dot{\bar{\theta}}_{11, k}\left\langle C p(\xi) L_{j}(\xi) L_{k}(\xi) L_{l}(\xi)\right\rangle \frac{1}{G R}
\end{aligned}
$$

and

$$
\begin{aligned}
& \ddot{\bar{x}}_{1}=-\frac{k_{x 1}}{M_{1}} \bar{x}_{1, l}+\frac{\sin \varphi_{1}}{M_{1}} K_{1}(t) \delta_{1, l} \\
& \ddot{\bar{x}}_{2}=-\frac{k_{x 2}}{M_{2}} \bar{x}_{2, l}-\frac{\sin \varphi_{1}}{M_{2}} K_{1}(t) \delta_{1, l}-K_{2}(t) \frac{\sin \varphi_{2}}{M_{2}} \delta_{2, l} \\
& \ddot{\bar{x}}_{3}=-\frac{k_{x 3}}{M_{3}} \bar{x}_{3, l}+K_{2}(t) \frac{\sin \varphi_{2}}{M_{3}} \delta_{2, l} \\
& \ddot{\bar{y}}_{1}=-\frac{k_{y 1}}{M_{1}} \bar{y}_{1, l}-\frac{\cos \varphi_{1}}{M_{1}} K_{1}(t) \delta_{1, l} \\
& \ddot{\bar{y}}_{2}=-\frac{k_{y 2}}{M_{2}} \bar{y}_{2, l}+\frac{\cos \varphi_{1}}{M_{2}} K_{1}(t) \delta_{1, l}+\frac{\cos \varphi_{2}}{M_{2}} K_{2}(t) \delta_{2, l} \\
& \ddot{\bar{y}}_{3}=-\frac{k_{y 3}}{M_{3}} \bar{y}_{3, l}-\frac{\cos \varphi_{2}}{M_{3}} K_{2}(t) \delta_{2, l}
\end{aligned}
$$

\section{Uncertainty in the dynamic response of the two stages gear system}

In this Section, the dynamic behaviour of the two stage gear transmission system is investigated. The sesults are presented using the polynomial chaos method. The PC results are compared with the results of the deterministic system derived in Section 5.

Figures 6 and 7 represent the mean value and standard deviation of the input angular displacement $\theta_{11}(t)$ and the linear displacement $x_{1}(t)$, respectively. The signal is random and it fluctuates around the boundary conditions (zero value). The standard deviation allows predicting the variation domain around the average value of the response. The mean value and the standard deviation of the dynamic displacement have the same order of amplitude. 

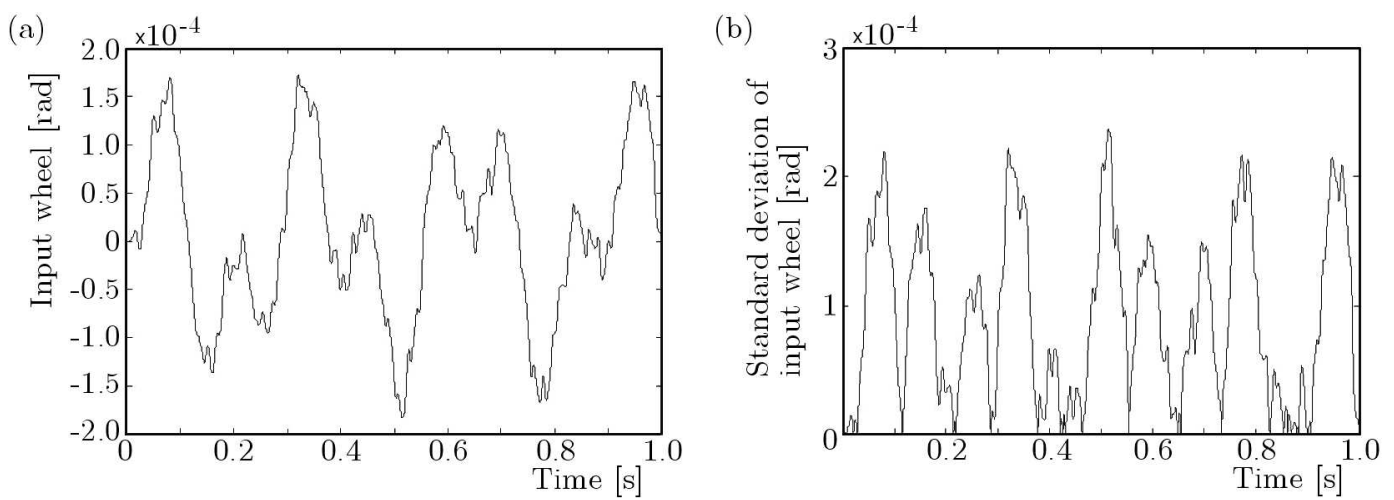

Fig. 6. Instantaneous mean value and standard deviation of $\theta_{11}(t)$; (a) mean value, (b) standard deviation
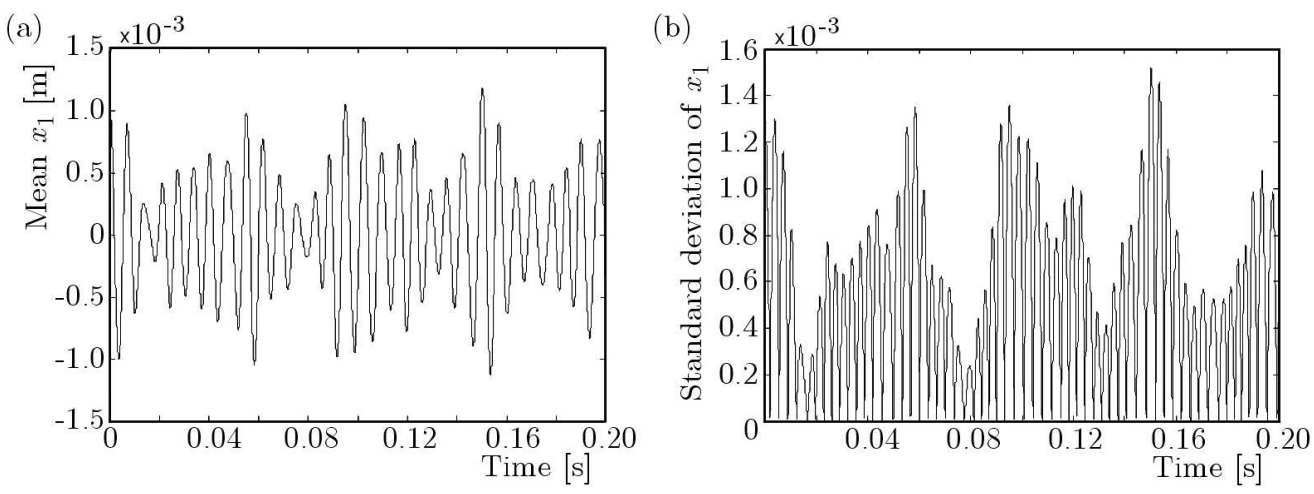

Fig. 7. Instantaneous mean value and standard deviation of $x_{1}(t)$; (a) mean value, (b) standard deviation

Figure 8 represents deflection of the first and the second tooth. The signal fluctuates around the zero value with an amplitude of the in order to $10^{-3}$. The signal is sinusoidal and have the same form of the deterministic model (Fig. 5), therefore, the polynomial chaos results provide a very good accuracy. In the case of uncertainty, at each time $t$, the performance coefficient varies randomly in the range of $[0.35,0.45]$. By contrast, it is constant in the case without uncertainty (deterministic model). So, there are many curves of teeth deflection relative to each performance coefficient. Here, Fig. 8, including Fig. 5, presents a more accurate range.
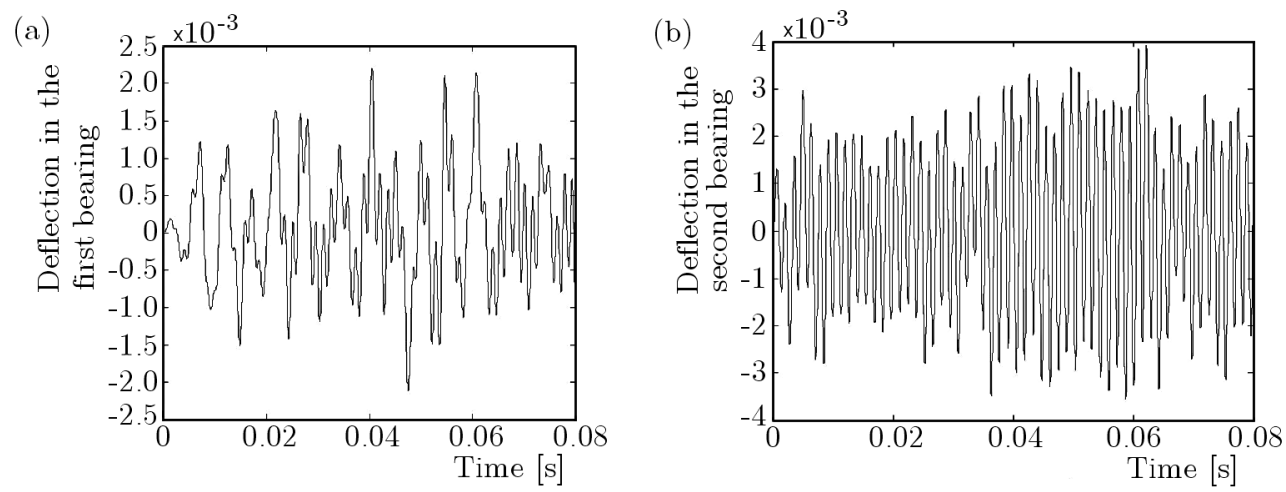

Fig. 8. Fluctuation of teeth deflection; (a) first bearing, (b) second bearing 
The fluctuation of the aerodynamic torque with consideration of the uncertainty according to the power coefficient is plotted in Fig. 9. The signal is sinusoidal and the amplitude is increasing between 0 and $60 \mathrm{Nm}$.

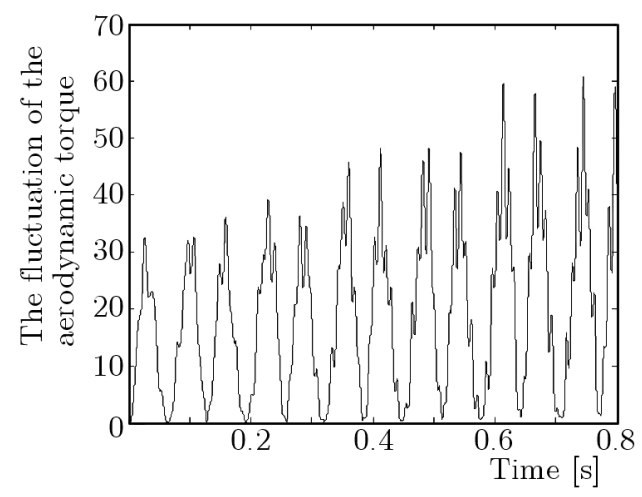

Fig. 9. Fluctuation of the aerodynamic torque

The results presented in Fig. 10 are found through orbits of the shaft. The orbits are constructed by using displacements in the $x$ - and $y$-directions. Figure 10 shows the evolution of orbits for the first and third bearing with a set of the random parameter defined previously. The bearings behave in an arbitrary way, which is not observed in the case in the model without uncertainty (Fig. 4).


Fig. 10. Evolutions of bearings displacements $y=f(x)$; (a) first bearing, (b) third bearing

\section{Conclusions}

The probabilistic dynamic response of a wind turbine system witha two-stage gearbox transmission system generated by an unceartain input aerodynamic torque has been incestigated. A new application of the polynomial chaos (PC) method is derived to study the influence of the input uncertainty parameter. The system structural dynamic response is presented using the polynomial chaos theory. Therefore, a set of mathematical equations is developed in order to predict the dynamic behavior of the two-stage spur gear system. Results of the uncertain model using the PC method are compared with the deterministic model.

The results suggest that the polynomial chaos method takes into account the uncertainty with a good efficiency. So, the PC approach can be considered as an efficient tool to take into account unceartianties in the study of dynamic behaviour of gearbox systems. 


\section{References}

1. Abboudi K., Walha L., Driss Y., Maatar M., Fakhfakh T., Haddar M., 2011, Dynamic behavior of a two-stage gear train used in a fixed-speed wind turbine, Journal of Mechanism and Machine Theory, 46

2. Beltran B., Benbouzid M.E.H., Mohamed-Ali T., 2011, Second-order sliding mode power control and grid fault-tolerance a dfig-based wind turbine, Revue des Sciences et de la Technologie, 2, 75-91

3. BuCKspan A., 2012, Nonlinear control of a wind turbine, Journal of Undergraduate Research, 13, $2,1-5$

4. Chantrasmi T., Constantine P., Etemadiz N., Iaccarino G., Wang Q., 2006, Uncertainty quantification in simple linear and non-linear problems, Annual Research Briefs

5. Fisher J., Bhattacharya R., 2008, Stability analysis of stochastic systems using polynomial chaos, American Control Conference, 4250-4255

6. Gebreslassie M.G., Tabor G.R., Belmont M.R., 2013, Numerical simulation of a new type of cross flow tidal turbine using OpenFOAM - Part I: Calibration of energy extraction, Renewable Energy, 50, 994-1004

7. Ghanem R., Spanos P.D., 1991, Stochastic Finite Elements. A Spectral Approach, SpringerVerlag, New York

8. Helsen J., Vanhollebeke F., Marrant B., Vandepitte D., Desmet W., 2011, Multibody modelling of varying complexity for modal behaviour analysis of wind turbine gearboxes, Renewable Energy, 36, 11, 3098-113

9. Isukapalli S.S., Roy A., Georgopoulos P.G., 1998a, Development and application of methods for assessing uncertainty in photochemical air quality problems, Interim Report for U.S.EPA National Exposure Research Laboratory

10. Isukapalli S.S., Roy A., Georgopoulos P.G., 1998b, Stochastic response surface methods (SRSMs) for uncertainty propagation: application to environmental and biological systems, Risk Analysis, 18, 351-363

11. Jakerman J.D., Roberts S.G., 2009, Stochastic Galerkin and collocation methods for quantifying uncertainties in differential equation, a review, ANZIAM Journal, 50, 815-830

12. Kalos M.H., Whitlock P.A., 1986, Monte Carlo ethods, Basics, Wiley-Interscience, 1, New York

13. Lei Y., Bai Y., Xu Z., Gao Q., Zhao C., 2013, An experimental investigation on aerodynamic performance of a coaxial rotor system with different rotor spacing and wind speed, Experimental Thermal and Fluid Science, 44, 779-785

14. Nechak L., Berger S., Aubry E., 2011, A polynomial chaos approach to the robust analysis of the dynamic behavior of friction systems, European Journal of Mechanics A/Solids, 594-607

15. Pettersson P., Iaccarino G., Nordstrom J., 2009, Numerical analysis of the Burgersequation in the presence of uncertainty, Journal of Computational Physics, 228, 8394-8412

16. Rubinstein R.Y., 1981, Simulation and the Monte Carlo Method, John Wiley \& Sons Inc. New York

17. Sandu A., Sandu C., Ahmadian M., 2006a, Modeling multibody dynamic systems with uncertainties. Part I: numerical application, Multibody System Dynamic, 15, 369-391

18. Sandu C., Sandu A., Ahmadian M., 2006b, Modeling multibody dynamic systems with uncertainties. Part II: theoretical and computational aspects, Multibody System Dynamic, 15, 241-262

19. Sloth C., Esbensen T., Stoustrup J., 2011, Robust and fault-tolerant linear parameter-varying control of wind turbines, Mechatronics, 21 
20. Wei S., Zhao J., Han Q., Chu F., 2015, Dynamic response analysis on torsional vibrations of wind turbine geared transmission system with uncertainty, Renewable Energy, 78, 60-67

21. Wiener N., 1938, The homogeneous chaos, American Journal of Mathematics, 60, 4, 897-936

22. Xiu D., Karniadakis G., 2002, The Wiener-Askey polynomial chaos for stochastic differential equations, SIAM Journal on Scientific Computing, 24, 2, 619-644

23. Zhu C., Xu X., Liu H., Luo T., Zhai H., 2014, Research on dynamical characteristics of wind turbine gearboxes with flexible pins, Renewable Energy, 68, 724-32

Manuscript received February 3, 2015; accepted for print October 15, 2015 